\title{
Synthesis of differentially protected ribitol derivatives from 3,4-O-benzylidene-D-ribono-1,5-lactone
}

\author{
Dirk J. Lefeber, ${ }^{\dagger}$ Peter Steunenberg, \\ Johannes F. G. Vliegenthart and Johannis P. Kamerling* \\ Bijvoet Center, Department of Bio-Organic Chemistry, Utrecht University, Padualaan 8, NL-3584 CH Utrecht, The Netherlands \\ Received 12 November 2004; accepted 10 December 2004
}

\begin{abstract}
Several differentially protected ribitol derivatives were synthesised using 3,4-O-benzylidene-D-ribono-1,5-lactone as versatile starting compounds for oligosaccharide synthesis. The obtained ribitol derivatives allow the regiospecific coupling of glycosyl donors to either of the hydroxyl groups of ribitol and can be applied for the preparation of polyhydroxylated compounds.

(c) 2004 Elsevier Ltd. All rights reserved.
\end{abstract}

\section{Introduction}

Ribitol is a common monosaccharide constituent of many bacterial capsular polysaccharides, such as those from Streptococcus pneumoniae types 6, 10A and 13, ${ }^{1}$ and from Haemophilus influenzae type b. ${ }^{2}$ Since in polysaccharides different hydroxyl groups of ribitol may be involved in the glycosidic linkage, regiospecific protection strategies are required for the synthesis of ribitolcontaining oligosaccharides. Furthermore, differently protected alditols are attractive synthetic intermediates for the preparation of various classes of polyhydroxylated compounds. ${ }^{3}$

In 1968 the product of the reaction of D-ribonolactone $\mathbf{1}$ (Scheme 1) with concentrated hydrochloric acid and benzaldehyde was postulated to be 3,5- $O$-benzylideneD-ribono-1,4-lactone $2{ }^{4}$ and as such it was used by others. ${ }^{5,6}$ However, in 1985 crystallographic studies of the acetylated compound showed the reaction product to be 3,4-O-benzylidene-D-ribono-1,5-lactone $\mathbf{3}$ instead of the 3,5-O-acetal. ${ }^{7}$ The use of the 1,5-lactone has been described in the preparation of $(2 R, 3 S, 4 R)$-dihydroxyproline $^{8}$ and pyrrolidine derivatives. ${ }^{9}$

In the context of our synthetic studies on ribitol-containing oligosaccharide fragments of bacterial polysaccharides, herein we report easily accessible protocols for the preparation of three differentially protected ribitol derivatives starting from 3,4- $O$-benzylidene-Dribono-1,5-lactone 3: 3,4-O-benzylidene-D-ribitol 4, 3,4-O-benzylidene-1-O-tert-butyldimethylsilyl-D-ribitol

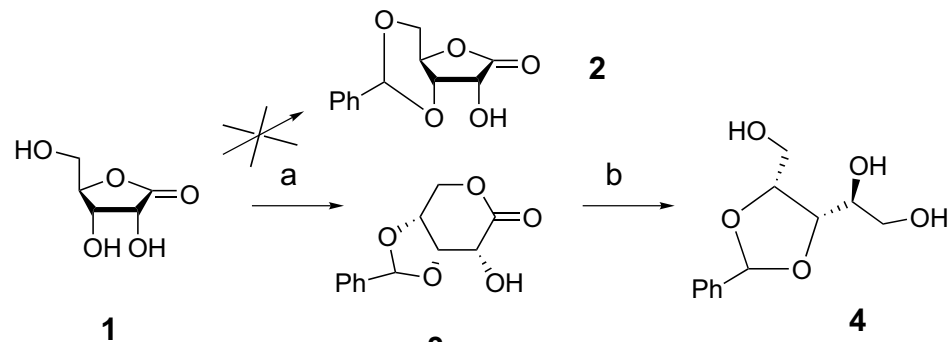

1

3

Scheme 1. Reagents and conditions: (a) $\mathrm{PhCHO}, \mathrm{HCl}, 87 \%$; (b) $\mathrm{NaBH}_{4}, \mathrm{MeOH}$, THF, $55^{\circ} \mathrm{C}, 85 \%$.

\footnotetext{
* Corresponding author. Tel.: +31 3025334 79; fax: +31 3025409 80; e-mail: j.p.kamerling@chem.uu.nl

${ }^{\dagger}$ Present address: Laboratory of Pediatrics and Neurology, Reinier Postlaan 4, 6525 GC Nijmegen, The Netherlands.
} 
6, and 3-O-allyl-2-O-tert-butyldimethylsilyl-5-O-tertbutyldiphenylsilyl-D-ribitol $\mathbf{1 0}$.

\section{Results and discussion}

Key compound 3,4-O-benzylidene-D-ribono-1,5-lactone 3 (Scheme 1) was prepared by reaction of D-ribono1,4-lactone 1 with benzaldehyde and concentrated $\mathrm{HCl}$ in $87 \%$ yield. ${ }^{1} \mathrm{H}$ NMR analysis of acetylated 3 showed a downfield shifted $\mathrm{H}-2\left(\delta 5.53 \mathrm{ppm}, \mathrm{d}, J_{2,3} 3.2 \mathrm{~Hz}\right)$, a benzylidene proton at $\delta 5.81 \mathrm{ppm}$, characteristic for an acetal proton in a 1,3-dioxolane ring system, and a ROESY contact between $\mathrm{H}-3$ and $\mathrm{H}-4$, in agreement with the orientation of $\mathrm{H}-3$ and $\mathrm{H}-4$ in compound 3 (cis), but not in compound 2. Opening and reduction of lactone 3 by using sodium borohydride and methanol in tetrahydrofuran afforded $\mathbf{4}$ in a yield of $85 \% .{ }^{1} \mathrm{H}$ NMR analysis of the acetylated product showed a downfield shifted H-2 ( $\delta 5.16 \mathrm{ppm}$, ddd), and an acetal proton at $\delta 5.84 \mathrm{ppm}$, which is evidence for a benzylidene group in a 5 -membered ring. Ribitol derivative $\mathbf{4}$ can be used for coupling at O-2 after protection of the primary hydroxyl groups, if no selectivity of the primary hydroxyl groups is required.

For the preparation of ribitol derivative 6 (Scheme 2), compound $\mathbf{3}$ was silylated with tert-butyldimethylsilyl (TBDMS) chloride ${ }^{10}$ to afford 5 in $95 \%$ yield. The position of the acetal proton in the ${ }^{1} \mathrm{H}$ NMR spectrum $(\delta$ $5.71 \mathrm{ppm}$ ) indicated a benzylidene group in a 1,3-dioxolane ring system, and the chemical shifts of C-3 and C-4 in the ${ }^{13} \mathrm{C}$ NMR spectrum (range $\delta \quad 73-77 \mathrm{ppm}^{8}$ ) indicated a 1,5-lactone. Reduction of $\mathbf{5}$ using sodium borohydride and methanol in tetrahydrofuran afforded $\mathbf{6}$ as the only product in $86 \%$ yield. The identity of $\mathbf{6}$ was derived from the ${ }^{1} \mathrm{H}$ NMR analysis of the acetylated product. First of all, two acetyl groups, a benzylidene group and a tert-butyldimethylsilyl group are present. A downfield shifted $\mathrm{H}-2$ was shown $(\delta 5.01 \mathrm{ppm}$, ddd $)$, which corresponds to an acetylated $\mathrm{O}-2$, thereby indicating the migration of the silyl group from $\mathrm{O}-2$ to $\mathrm{O}-1$. Furthermore, a comparison of the H-1 chemical shifts in acetylated $\mathbf{6}$ with acetylated 4 (Scheme 1) showed an upfield shift from $>4.10$ to 3.93 and $3.84 \mathrm{ppm}$, supporting that $\mathrm{O}-1$ is not acetylated in $\mathbf{6}$. The position of the acetal proton at $\delta 5.84 \mathrm{ppm}$ indicated that the 1,3-dioxolane ring system was intact. A similar silyl migration under reducing conditions with sodium borohydride has been observed before. ${ }^{11}$ Thus, due to a facile silyl migration, ribitol derivative $\mathbf{6}$ is obtained, which can be used for reactions at O-2 with the option of selective protection of the primary hydroxyl functions.

The synthesis of a ribitol derivative in which O-3 and O-4 are selectively accessible, was started from D-ribonolactone derivative 5 (Scheme 3). Debenzylidenation of $\mathbf{5}$ without affecting the silyl group was accomplished by catalytic hydrogenation using a palladium catalyst in ethyl acetate, affording 7 in $97 \%$ yield. It should be noted that hydrogenation of $\mathbf{5}$ in ethanol led to cleavage of the silyl group. The cleavage of a tert-butyldimethylsilyl group has been described before during a transfer hydrogenation in methanol. ${ }^{12}$ The identity of 7 was confirmed by NMR analysis. The position of $\mathrm{C}-4$ in the ${ }^{13} \mathrm{C}$ NMR spectrum of 7 ( $\delta 84.6 \mathrm{ppm})$ is characteristic for a 1,4-lactone; the ${ }^{1} \mathrm{H}$ NMR spectrum of acetylated 7 showed a downfield shifted H-3 $(\delta 5.31 \mathrm{ppm}$, dd $)$ and a non- $O$ acetylated O-4 $(\delta 4.65 \mathrm{ppm}, \mathrm{m})$. A similar rearrangement of a ribono-1,5-lactone to a ribono-1,4-lactone after debenzylidenation with trifluoroacetic acid had been found previously. ${ }^{8}$ For the selective protection of O-5 in 7, the tert-butyldiphenylsilyl (TBDPS) group was chosen, ${ }^{13}$ and reaction of 7 with tert-butyldiphenylsilyl

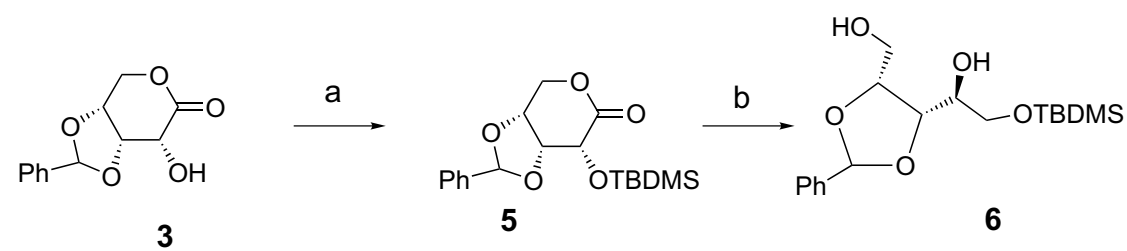

Scheme 2. Reagents and conditions: (a) TBDMSCl, $\mathrm{C}_{5} \mathrm{H}_{5} \mathrm{~N}$, DMAP, $95 \%$; (b) $\mathrm{NaBH}_{4}, \mathrm{MeOH}$, THF, $55^{\circ} \mathrm{C}, 86 \%$.
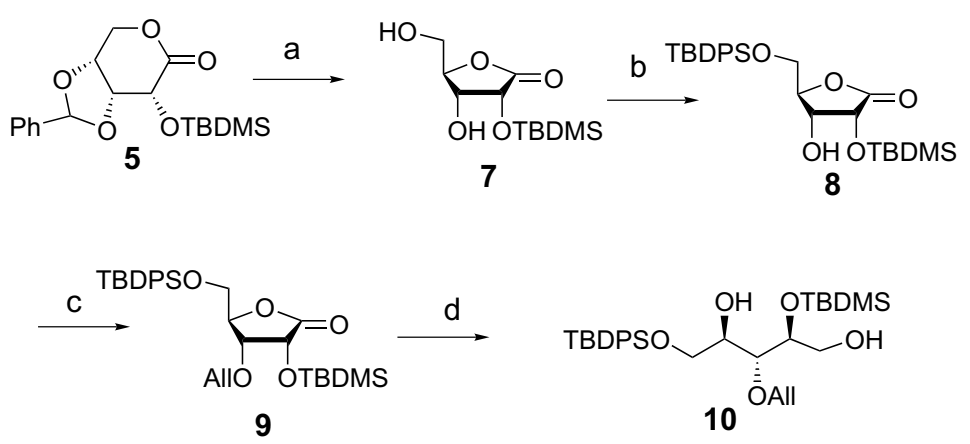

Scheme 3. Reagents and conditions: (a) $\mathrm{H}_{2}, 10 \% \mathrm{Pd} / \mathrm{C}$, EtOAc, $97 \%$; (b) TBDPSCl, $\mathrm{CH}_{2} \mathrm{Cl}_{2}, \mathrm{C}_{5} \mathrm{H}_{5} \mathrm{~N}, \mathrm{DMAP}, 80 \%$; (c) (i) $\mathrm{AocCl}, \mathrm{CH}_{2} \mathrm{Cl}_{2}, \mathrm{C}_{5} \mathrm{H}_{5} \mathrm{~N}$, $0{ }^{\circ} \mathrm{C}, 84 \%$, (ii) $\left(\mathrm{PPh}_{3}\right)_{4} \mathrm{Pd}(0), \mathrm{CH}_{3} \mathrm{CN}, \mathrm{He}, 55^{\circ} \mathrm{C}, 75 \%$; (d) $\mathrm{NaBH}_{4}, \mathrm{MeOH}, \mathrm{THF}, 55^{\circ} \mathrm{C}, 86 \%$. 
chloride ${ }^{14}$ gave $\mathbf{8}$ in $80 \%$ yield. The remaining hydroxyl function at C-3 was chosen to be allylated. Sodium hydride-mediated allylation of $\mathbf{8}$ turned out to be impossible due to opening of the base-labile lactone. Therefore, a two-step allylation procedure was used..$^{15}$ Allyloxycarbonylation and subsequent decarboxylation using tetrakis-triphenylphosphine-palladium(0) gave 9 in 63\% yield. Reductive opening of the lactone using sodium borohydride and methanol in tetrahydrofuran afforded the ribitol derivative $\mathbf{1 0}$ in a yield of $86 \%$. The ${ }^{1} \mathrm{H}$ NMR spectrum of acetylated $\mathbf{1 0}$ showed a downfield shifted H-4 $(\delta 5.23, \mathrm{dt})$, and the presence of two acetyl groups, a TBDMS group, a TBDPS group and an allyl group. The resulting ribitol derivative $\mathbf{1 0}$ allows the glycosidic coupling to either of the skeleton oxygen atoms.

\section{Conclusion}

3,4-O-Benzylidene-D-ribono-1,5-lactone has been shown to be a versatile compound for the preparation of several differentially protected ribitol derivatives. These products can be used for the regiospecific coupling of protected sugar donors to one of the hydroxyl groups of ribitol or for the preparation of polyhydroxylated compounds.

\section{Experimental}

\subsection{General methods}

All reagents were used as obtained commercially, without any further purification. Reactions were monitored by TLC on Kieselgel $60 \mathrm{~F}_{254}$ (Merck); compounds were visualised, after examination under UV light, by charring with phenol-sulfuric acid or $\mathrm{KMnO}_{4}$ and $\mathrm{Na}_{2} \mathrm{CO}_{3}$ in water. In the work-up procedures of reaction mixtures, organic solutions were washed with appropriate amounts of the indicated aqueous solutions, then dried over $\mathrm{MgSO}_{4}$ and concentrated under reduced pressure at $20-40{ }^{\circ} \mathrm{C}$ on a water bath. Column chromatography was performed on Kieselgel $60 \mathrm{~F}_{254}$ (Merck, 70-230 mesh). Optical rotations were measured in $\mathrm{CHCl}_{3}$ with a Perkin-Elmer 241 polarimeter, using a $10-\mathrm{cm} 1-\mathrm{mL}$ cell. ${ }^{1} \mathrm{H}$ NMR spectra were recorded at $27^{\circ} \mathrm{C}$ with a Bruker AC 300 spectrometer; the values of $\delta_{\mathrm{H}}$ are given in ppm relative to the signal for internal $\mathrm{Me}_{4} \mathrm{Si}(\delta 0)$ for solutions in $\mathrm{CDCl}_{3} \cdot{ }^{13} \mathrm{C}$ (APT, $75 \mathrm{MHz}$ ) NMR spectra were recorded at $27^{\circ} \mathrm{C}$ with a Bruker AC 300 spectrometer; indicated ppm values for $\delta_{\mathrm{C}}$ are relative to the signal of $\mathrm{CDCl}_{3}\left(\delta\right.$ 76.9) for solutions in $\mathrm{CDCl}_{3}$. Twodimensional ${ }^{1} \mathrm{H}-{ }^{1} \mathrm{H}$ correlation spectra (TOCSY and ROESY) were recorded using a Bruker AMX 500 apparatus $(500 \mathrm{MHz})$ at $27^{\circ} \mathrm{C}$. Elemental analyses were carried out by $\mathrm{H}$. Kolbe Mikroanalytisches Laboratorium (Mülheim an der Ruhr, Germany).

\subsection{3,4-O-Benzylidene-D-ribitol, 4}

To a solution of D-ribono-1,4-lactone 1 (5.56 g, $38 \mathrm{mmol})$ in benzaldehyde $(50 \mathrm{~mL})$ was added concentrated $\mathrm{HCl}(5 \mathrm{~mL})$. After $7 \mathrm{~h}, \mathrm{Et}_{2} \mathrm{O}(60 \mathrm{~mL})$ was added, and the precipitate collected by filtration. The residue was washed with aq $10 \% \mathrm{NaHCO}_{3}$ and water, and recrystallised from refluxing acetone, yielding $3(7.75 \mathrm{~g}$, $87 \%) .{ }^{1} \mathrm{H}$ NMR (DMSO- $\left.d_{6}\right): \delta 7.46-7.32(\mathrm{~m}, 5 \mathrm{H}$, $P h \mathrm{CH}), 5.74(\mathrm{~s}, 1 \mathrm{H}, \mathrm{PhCH}), 4.69\left(\mathrm{dd}, 1 \mathrm{H}, J_{3,4} 8.0, J_{2,3}\right.$ $3.2 \mathrm{~Hz}, \mathrm{H}-3$ ), 4.64 (br d, $\left.1 \mathrm{H}, J_{4,5 \mathrm{~b}} 1.6 \mathrm{~Hz}, \mathrm{H}-4\right), 4.62$ $(\mathrm{d}, 1 \mathrm{H}, \mathrm{H}-2), 4.42\left(\mathrm{dd}, 1 \mathrm{H}, J_{5 \mathrm{a}, 5 \mathrm{~b}} 13.1 \mathrm{~Hz}, \mathrm{H}-5 \mathrm{~b}\right), 4.32$ (d, $1 \mathrm{H}, \mathrm{H}-5 \mathrm{a}) ;{ }^{1} \mathrm{H}$ NMR $\left(\mathrm{CDCl}_{3}+\mathrm{MeOH}-d_{3}\right): \delta 7.47-$ $7.39(\mathrm{~m}, 5 \mathrm{H}, P h \mathrm{CH}), 5.81(\mathrm{~s}, 1 \mathrm{H}, \mathrm{PhCH}), 4.84(\mathrm{dd}$, $\left.1 \mathrm{H}, J_{3,4} 8.1, J_{2,3} 3.3 \mathrm{~Hz}, \mathrm{H}-3\right), 4.69(\mathrm{~m}, 1 \mathrm{H}, \mathrm{H}-4), 4.56$ (d, $1 \mathrm{H}, \mathrm{H}-2), 4.39$ (dd, $\left.1 \mathrm{H}, J_{5 \mathrm{a}, 5 \mathrm{~b}} 13.2 \mathrm{~Hz}, \mathrm{H}-5\right)$. For further analysis, a small amount of $\mathbf{3}$ was acetylated with $\mathrm{Ac}_{2} \mathrm{O}$ in pyridine. ${ }^{1} \mathrm{H}$ NMR $\left(\mathrm{CDCl}_{3}\right): \delta 7.47-7.39(\mathrm{~m}$, $5 \mathrm{H}, P h \mathrm{CH}), 5.81(\mathrm{~s}, 1 \mathrm{H}, \mathrm{PhCH}), 5.53\left(\mathrm{~d}, 1 \mathrm{H}, J_{2,3}\right.$ $3.2 \mathrm{~Hz}, \mathrm{H}-2), 4.84$ (dd, 1H, $J_{3.4} 8.0 \mathrm{~Hz}, \mathrm{H}-3$ ), 4.69 (br d, $1 \mathrm{H}, \mathrm{H}-4), 4.59\left(\mathrm{~d}, 1 \mathrm{H}, J_{5 \mathrm{a}, 5 \mathrm{~b}} 13.4 \mathrm{~Hz}, \mathrm{H}-5 \mathrm{a}\right), 4.38$ (dd, $\left.1 \mathrm{H}, J_{4,5 \mathrm{~b}} 1.7 \mathrm{~Hz}, \mathrm{H}-5 \mathrm{~b}\right), 2.25$ (s, 3H, $\mathrm{CH}_{3} \mathrm{CO}$ ).

To a solution of 3 (200 mg, $0.85 \mathrm{mmol})$ in THF (2.5 mL) was added $\mathrm{NaBH}_{4}(80 \mathrm{mg})$. The mixture was heated to $55^{\circ} \mathrm{C}$, and $\mathrm{MeOH}(0.8 \mathrm{~mL})$ was added dropwise over $30 \mathrm{~min}$. After $1 \mathrm{~h}$, TLC (95:5 $\left.\mathrm{CH}_{2} \mathrm{Cl}_{2}-\mathrm{MeOH}\right)$ showed complete conversion of 3 into $4\left(R_{\mathrm{f}} 0.45\right)$. Then, the mixture was quenched by addition of HOAc, and coconcentrated with $\mathrm{MeOH}$. The crude product was purified by column chromatography $\left(98: 2 \mathrm{CH}_{2} \mathrm{Cl}_{2}-\mathrm{MeOH}\right)$ to yield $4(170 \mathrm{mg}, 85 \%)$. For analysis, a small aliquot was acetylated with $\mathrm{Ac}_{2} \mathrm{O}$ in pyridine. ${ }^{1} \mathrm{H}$ NMR $\left(\mathrm{CDCl}_{3}\right): \delta 7.49$ 7.37 (m, 5H, PhCH), 5.84 (s, 1H, $\mathrm{PhCH}$ ), 5.16 (ddd, 1H, $\mathrm{H}-2), 2.08,2.07$ and $2.06\left(3 \mathrm{~s}\right.$, each $\left.3 \mathrm{H}, \mathrm{CH}_{3} \mathrm{CO}\right)$.

\subsection{3,4-O-Benzylidene-2-O-tert-butyldimethylsilyl-D- ribono-1,5-lactone, 5}

To a solution of $3(7.75 \mathrm{~g}, 33 \mathrm{mmol})$ in pyridine $(60 \mathrm{~mL})$ at $0{ }^{\circ} \mathrm{C}$ were added TBDMSCl $(7.7 \mathrm{~g}, 50 \mathrm{mmol})$ and a catalytic amount of DMAP. The mixture was allowed to reach room temperature, and after $20 \mathrm{~h}$, a second portion of TBDMSCl $(2.0 \mathrm{~g}, 13 \mathrm{mmol})$ was added. After $25 \mathrm{~h}$, TLC (95:5 $\left.\mathrm{CH}_{2} \mathrm{Cl}_{2}-\mathrm{MeOH}\right)$ showed complete conversion of 3 into 5. The mixture was diluted with EtOAc, washed with aq $10 \% \mathrm{NaHCO}_{3}$ and aq $10 \% \mathrm{NaCl}$, and the organic layer dried, filtered and concentrated. Column chromatography ( $7: 3$ toluene-EtOAc) of the residue afforded $\mathbf{5}$ as a white solid $(11.0 \mathrm{~g}, 95 \%)$. $[\alpha]_{\mathrm{D}}=-134\left(c \quad 0.9, \mathrm{CHCl}_{3}\right) ; R_{\mathrm{f}} 0.89\left(95: 5 \mathrm{CH}_{2} \mathrm{Cl}_{2}\right.$ $\mathrm{MeOH}) ;{ }^{1} \mathrm{H}$ NMR $\left(\mathrm{CDCl}_{3}\right): \delta \quad 7.47-7.33(\mathrm{~m}, 5 \mathrm{H}$, $P h \mathrm{CH}), 5.71(\mathrm{~s}, 1 \mathrm{H}, \mathrm{PhCH}), 4.64\left(\mathrm{dd}, 1 \mathrm{H}, J_{2,3} 3.2, J_{3,4}\right.$ $8.0 \mathrm{~Hz}, \mathrm{H}-3), 4.51$ (dd, $\left.1 \mathrm{H}, J_{4,5 \mathrm{~b}} 1.4 \mathrm{~Hz}, \mathrm{H}-4\right), 4.45$ (d, $1 \mathrm{H}, \mathrm{H}-2), 4.36$ (d, $\left.1 \mathrm{H}, J_{5 \mathrm{a}, 5 \mathrm{~b}} 13.4 \mathrm{~Hz}, \mathrm{H}-5 \mathrm{a}\right), 4.15$ (dd, $1 \mathrm{H}, \mathrm{H}-5 \mathrm{~b}), 0.94\left(\mathrm{~s}, 9 \mathrm{H}, \mathrm{SiC}\left(\mathrm{CH}_{3}\right)_{3}\right), 0.20$ and $0.13(2 \mathrm{~s}$, each $\left.3 \mathrm{H}, \mathrm{Si}\left(\mathrm{CH}_{3}\right)_{2}\right) ;{ }^{13} \mathrm{C}$ NMR $\left(\mathrm{CDCl}_{3}\right): \delta 169.4(\mathrm{C}-1)$, 135.1, 129.8, 128.2 and 127.2 $(\mathrm{PhCH}), 104.1(\mathrm{PhCH})$, 77.3, 73.1 and $69.6(\mathrm{C}-2, \mathrm{C}-3, \mathrm{C}-4), 67.0(\mathrm{C}-5), 25.6$ $\left(\mathrm{SiC}\left(\mathrm{CH}_{3}\right)_{3}\right), 18.2 \quad\left(\mathrm{SiC}\left(\mathrm{CH}_{3}\right)_{3}\right)$. Anal. Calcd for $\mathrm{C}_{18} \mathrm{H}_{26} \mathrm{O}_{5} \mathrm{Si}$ (350.5): C, 61.69; H, 7.48. Found: C, $61.80 ; \mathrm{H}, 7.47$.

\subsection{3,4-O-Benzylidene-1-O-tert-butyldimethylsilyl-D- ribitol, 6}

To a solution of 5 (491 mg, $1.4 \mathrm{mmol})$ in dry THF $(5 \mathrm{~mL})$ was added $\mathrm{NaBH}_{4}(130 \mathrm{mg})$. The mixture was 
heated to $55^{\circ} \mathrm{C}$, and $\mathrm{MeOH}$ added dropwise over $30 \mathrm{~min}$. After $1 \mathrm{~h}$, TLC (95:5 $\left.\mathrm{CH}_{2} \mathrm{Cl}_{2}-\mathrm{MeOH}\right)$ showed complete conversion of 5 into $\mathbf{6}\left(R_{\mathrm{f}} 0.76\right)$. Then, the mixture was quenched with HOAc and coconcentrated with $\mathrm{MeOH}$ and toluene. The residue was purified by column chromatography (7:3 toluene-EtOAc) to yield $\mathbf{6}$ (400 mg, 86\%). For analysis, a small amount of 6 was acetylated with $\mathrm{Ac}_{2} \mathrm{O}$ in pyridine. ${ }^{1} \mathrm{H}$ NMR $\left(\mathrm{CDCl}_{3}\right): \delta$ 7.48-7.37 (m, 5H,PhCH), $5.84(\mathrm{~s}, 1 \mathrm{H}, \mathrm{PhCH}), 5.01$ (ddd, $\left.1 \mathrm{H}, J_{2,3} 8.3, J_{1 \mathrm{a}, 2} 2.7, J_{1 \mathrm{~b}, 2} 3.8 \mathrm{~Hz}, \mathrm{H}-2\right), 4.52$ (dd, $\left.1 \mathrm{H}, J_{3,4} 6.1 \mathrm{~Hz}, \mathrm{H}-3\right), 4.49-4.42(\mathrm{~m}, 2 \mathrm{H}, \mathrm{H}-4$ and H-5), 4.08 (dd, 1H, $\left.J_{5 \mathrm{a}, 5 \mathrm{~b}} 12.8 \mathrm{~Hz}, \mathrm{H}-5\right), 3.93$ (dd, $1 \mathrm{H}$, $\left.J_{1 \mathrm{a}, 1 \mathrm{~b}} 11.5 \mathrm{~Hz}, \mathrm{H}-1 \mathrm{a}\right), 3.84$ (dd, 1H, H-1b), 2.09 and $2.05\left(2 \mathrm{~s}\right.$, each $\left.3 \mathrm{H}, \mathrm{CH}_{3} \mathrm{CO}\right), 0.88\left(\mathrm{~s}, 9 \mathrm{H}, \mathrm{SiC}\left(\mathrm{CH}_{3}\right)_{3}\right)$, $0.03\left(\mathrm{~s}, 6 \mathrm{H}, \mathrm{Si}\left(\mathrm{CH}_{3}\right)_{2}\right)$.

\subsection{2-O-tert-Butyldimethylsilyl-D-ribono-1,4-lactone, 7}

A solution of $5(10.8 \mathrm{~g}, 31 \mathrm{mmol})$ in EtOAc $(150 \mathrm{~mL})$, containing $10 \% \mathrm{Pd} / \mathrm{C}(2.5 \mathrm{~g})$, was hydrogenated for $6 \mathrm{~h}$, then the mixture filtered over Celite and concentrated. The residue was dissolved in EtOAc $(150 \mathrm{~mL})$, $10 \% \mathrm{Pd} / \mathrm{C}(1.5 \mathrm{~g})$ was added and the suspension subjected to a second hydrogenation. After $2 \mathrm{~h}$, the mixture was filtered over Celite and concentrated. The crude product was purified by column chromatography (95:5 $\left.\mathrm{CH}_{2} \mathrm{Cl}_{2}-\mathrm{MeOH}\right)$ to obtain 7 as a white solid $(8.0 \mathrm{~g}$, $97 \%) . \quad[\alpha]_{\mathrm{D}}=+24 \quad(c \quad 1.4, \quad \mathrm{MeOH}) ; \quad R_{\mathrm{f}} 0.64 \quad(95: 5$ $\left.\mathrm{CH}_{2} \mathrm{Cl}_{2}-\mathrm{MeOH}\right) ;{ }^{1} \mathrm{H}$ NMR $\left(\mathrm{CDCl}_{3}\right): \delta 4.68(\mathrm{~d}, 1 \mathrm{H}$, $\left.J_{2,3} 5.5 \mathrm{~Hz}, \mathrm{H}-2\right), 4.47\left(\mathrm{~m}, 1 \mathrm{H}, J_{3,4} 0.9, J_{4,5 \mathrm{a}} 2.4, J_{4,5 \mathrm{~b}}\right.$ $2.6 \mathrm{~Hz}, \mathrm{H}-4), 4.31$ (ddd, $\left.1 \mathrm{H}, J_{3, \mathrm{OH}} 1.8 \mathrm{~Hz}, \mathrm{H}-3\right), 3.97$ (ddd, $\left.1 \mathrm{H}, J_{5 \mathrm{a}, 5 \mathrm{~b}} 12.6, \mathrm{H}-5 \mathrm{~b}\right), 3.79$ (ddd, 1H, H-5a), $0.94\left(\mathrm{~s}, 9 \mathrm{H}, \mathrm{SiC}\left(\mathrm{CH}_{3}\right)_{3}\right), 0.23$ and $0.20(2 \mathrm{~s}$, each $3 \mathrm{H}$, $\left.\mathrm{Si}\left(\mathrm{CH}_{3}\right)_{2}\right) ;{ }^{13} \mathrm{C} \mathrm{NMR}\left(\mathrm{CDCl}_{3}\right): \delta 170.5(\mathrm{C}-1), 84.6$, 70.4 and 69.9 (C-2, C-3, C-4), 61.6 (C-5), 25.5 $\left(\mathrm{SiC}\left(\mathrm{CH}_{3}\right)_{3}\right), \quad 18.1 \quad\left(\mathrm{SiC}\left(\mathrm{CH}_{3}\right)_{3}\right)$. Anal. Calcd for $\mathrm{C}_{11} \mathrm{H}_{22} \mathrm{O}_{5} \mathrm{Si}$ (262.4): C, 50.36; $\mathrm{H}$, 8.45. Found: $\mathrm{C}$, $51.43 ; \mathrm{H}, 8.53$. For further analysis, a small amount of 7 was acetylated with $\mathrm{Ac}_{2} \mathrm{O}$ in pyridine. ${ }^{1} \mathrm{H}$ NMR $\left(\mathrm{CDCl}_{3}\right): \delta 5.31\left(\mathrm{dd}, 1 \mathrm{H}, J_{2,3} 5.9, J_{3,4} 2.0 \mathrm{~Hz}, \mathrm{H}-3\right)$, $4.64\left(\mathrm{~m}, 1 \mathrm{H}, J_{4,5 \mathrm{a}} 3.4, J_{4,5 \mathrm{~b}} 3.9 \mathrm{~Hz}, \mathrm{H}-4\right), 4.59(\mathrm{~d}, 1 \mathrm{H}$, $\mathrm{H}-2), 4.38\left(\mathrm{dd}, 1 \mathrm{H}, J_{5 \mathrm{a}, 5 \mathrm{~b}} 12.6 \mathrm{~Hz}, \mathrm{H}-5 \mathrm{a}\right), 4.24(\mathrm{dd}, 1 \mathrm{H}$, $\mathrm{H}-5 \mathrm{~b}), 2.12$ and $2.10\left(2 \mathrm{~s}\right.$, each $\left.3 \mathrm{H}, \mathrm{CH}_{3} \mathrm{CO}\right), 0.91$ (s, $\left.9 \mathrm{H}, \mathrm{SiC}\left(\mathrm{CH}_{3}\right)_{3}\right), 0.20$ and $0.13\left(2 \mathrm{~s}\right.$, each $\left.3 \mathrm{H}, \mathrm{Si}\left(\mathrm{CH}_{3}\right)_{2}\right)$.

\subsection{2-O-tert-Butyldimethylsilyl-5-O-tert-butyldiphenyl- silyl-D-ribono-1,4-lactone, 8}

To a solution of $7(3.5 \mathrm{~g}, 14 \mathrm{mmol})$ in $\mathrm{CH}_{2} \mathrm{Cl}_{2}(66 \mathrm{~mL})$ and pyridine $(4.4 \mathrm{~mL})$ were added TBDPSCl $(4.1 \mathrm{~mL}$, $15 \mathrm{mmol})$ and $\mathrm{Et}_{3} \mathrm{~N}(1.0 \mathrm{~mL})$. After $16 \mathrm{~h}$, the mixture was quenched with ice, diluted with $\mathrm{CH}_{2} \mathrm{Cl}_{2}$, washed with $10 \%$ aq $\mathrm{NaHCO}_{3}$, dried, filtered and concentrated. The residue was purified by column chromatography (9:1 toluene-EtOAc) to afford $8(5.4 \mathrm{~g}, \quad 80 \%)$. $[\alpha]_{\mathrm{D}}=+17\left(c 1, \mathrm{CHCl}_{3}\right) ; R_{\mathrm{f}} 0.54(9: 1$ toluene-EtOAc); ${ }^{1} \mathrm{H}$ NMR $\left(\mathrm{CDCl}_{3}\right): \delta 7.71-7.67,7.39-7.31(2 \mathrm{~m}, 10 \mathrm{H}$, $P h_{2} \mathrm{Si}$ ), 4.82 (d, $\left.1 \mathrm{H}, J_{2,3} 5.6 \mathrm{~Hz}, \mathrm{H}-2\right), 4.34\left(\mathrm{~m}, 1 \mathrm{H} J_{3,4}\right.$ $\left.1.2, J_{4,5 \mathrm{a}} 1.7, J_{4,5 \mathrm{~b}} 2.4 \mathrm{~Hz}, \mathrm{H}-4\right), 4.28$ (dd, $1 \mathrm{H}, \mathrm{H}-3$ ), $3.82\left(\mathrm{dd}, 1 \mathrm{H}, J_{5 \mathrm{a}, 5 \mathrm{~b}} 11.8 \mathrm{~Hz}, \mathrm{H}-5 \mathrm{a}\right), 3.64(\mathrm{dd}, 1 \mathrm{H}$, $\mathrm{H}-5 \mathrm{~b}), \quad 1.04 \quad\left(\mathrm{~s}, \quad 9 \mathrm{H}, \quad(\mathrm{Ph})_{2} \mathrm{SiC}\left(\mathrm{CH}_{3}\right)_{3}\right), \quad 0.93 \quad(\mathrm{~s}$, $\left.9 \mathrm{H}, \quad\left(\mathrm{CH}_{3}\right)_{2} \mathrm{SiC}\left(\mathrm{CH}_{3}\right)_{3}\right), \quad 0.23$ and $0.17(2 \mathrm{~s}$, each $\left.3 \mathrm{H}, \mathrm{Si}\left(\mathrm{CH}_{3}\right)_{2}\right)$; ${ }^{13} \mathrm{C}$ NMR $\left(\mathrm{CDCl}_{3}\right): \delta 174.4(\mathrm{C}-1)$,
135.4-127.5 $\left((\mathrm{Ph})_{2} \mathrm{SiC}\left(\mathrm{CH}_{3}\right)_{3}\right), \quad 84.2, \quad 70.3$ and 69.9 (C-2, C-3, C-4), $63.4(\mathrm{C}-5), 26.5\left((\mathrm{Ph})_{2} \mathrm{SiC}\left(\mathrm{CH}_{3}\right)_{3}\right)$, $25.2\left(\left(\mathrm{CH}_{3}\right)_{2} \mathrm{SiC}\left(\mathrm{CH}_{3}\right)_{3}\right), 18.8\left((\mathrm{Ph})_{2} \mathrm{SiC}\left(\mathrm{CH}_{3}\right)_{3}\right), 18.1$ $\left(\left(\mathrm{CH}_{3}\right)_{2} \mathrm{SiC}\left(\mathrm{CH}_{3}\right)_{3}\right)$. Anal. Calcd for $\mathrm{C}_{27} \mathrm{H}_{40} \mathrm{O}_{5} \mathrm{Si}_{2}$ (500.8): C, 64.76; H, 8.05. Found: C, 65.01; H, 7.98.

\subsection{3-O-Allyl-2-O-tert-butyldimethylsilyl-5-O-tert-butyl- diphenylsilyl-D-ribono-1,4-lactone, 9}

A stirred solution of $8(3.5 \mathrm{~g}, 7.0 \mathrm{mmol})$ in dry acetonitrile $(3.3 \mathrm{~mL})$ and dry pyridine $(1.1 \mathrm{~mL})$ was cooled to $0{ }^{\circ} \mathrm{C}$. Allyl chloroformate $(1.46 \mathrm{~mL}, 14 \mathrm{mmol})$ in dry acetonitrile $(3.3 \mathrm{~mL})$ was added dropwise over $45 \mathrm{~min}$, and stirring at $0{ }^{\circ} \mathrm{C}$ continued for $1 \mathrm{~h}$. Then, TLC (9:1 toluene-EtOAc) showed complete conversion of $\mathbf{8}$ into $9\left(R_{\mathrm{f}} 0.83\right)$. After hydrolysing the remaining allyl chloroformate by addition of ice, the mixture was diluted with EtOAc, washed with water, dried, filtered and concentrated. The residue was purified by column chromatography (toluene) $(3.4 \mathrm{~g}, 84 \%)$. $[\alpha]_{\mathrm{D}}=+27\left(c 1, \mathrm{CHCl}_{3}\right)$; ${ }^{\mathrm{T}} \mathrm{H}$ NMR $\left(\mathrm{CDCl}_{3}\right): \delta 7.66-7.61,7.44-7.38(2 \mathrm{~m}, 10 \mathrm{H}$, $\mathrm{Ph}_{2} \mathrm{Si}$ ), 5.97-5.84 (m, $1 \mathrm{H}, \mathrm{CH}_{2}-\mathrm{CH}=\mathrm{CH}_{2}$ ), 5.39 (br d, $\left.1 \mathrm{H}, J_{2,3} 6.0, J_{3,4}<1.0 \mathrm{~Hz}, \mathrm{H}-3\right), 5.38-5.31,5.27-5.22$ $\left(2 \mathrm{~m}\right.$, each $\left.1 \mathrm{H}, \mathrm{CH}_{2}-\mathrm{CH}=\mathrm{CH}_{2}\right), 4.88(\mathrm{~d}, 1 \mathrm{H}, \mathrm{H}-2)$, 4.69-4.56 (m, $\left.2 \mathrm{H}, \mathrm{CH}_{2}-\mathrm{CH}=\mathrm{CH}_{2}\right), 4.47\left(\mathrm{~m}, 1 \mathrm{H}, J_{4,5 \mathrm{a}}\right.$ $\left.1.8, J_{4,5 \mathrm{~b}} 2.5 \mathrm{~Hz}, \mathrm{H}-4\right), 3.91\left(\mathrm{dd}, 1 \mathrm{H}, J_{5 \mathrm{a}, 5 \mathrm{~b}} 11.8 \mathrm{~Hz}, \mathrm{H}-\right.$ 5b), 3.80 (dd, 1H, H-5a), 1.06 (s, 9H, $\left.(\mathrm{Ph})_{2} \mathrm{SiC}\left(\mathrm{CH}_{3}\right)_{3}\right)$, $0.93\left(\mathrm{~s}, 9 \mathrm{H},\left(\mathrm{CH}_{3}\right)_{2} \mathrm{SiC}\left(\mathrm{CH}_{3}\right)_{3}\right), 0.21$ and $0.14(2 \mathrm{~s}$, each $\left.3 \mathrm{H}, \mathrm{Si}\left(\mathrm{CH}_{3}\right)_{2}\right) ;{ }^{13} \mathrm{C}$ NMR $\left(\mathrm{CDCl}_{3}\right): \delta 173.5(\mathrm{C}-1), 153.8$ $\left(\mathrm{CO}-\mathrm{CH}_{2}-\mathrm{CH}=\mathrm{CH}_{2}\right), \quad 135.3-127.8 \quad\left((\mathrm{Ph})_{2} \mathrm{SiC}\left(\mathrm{CH}_{3}\right)_{3}\right)$, $119.1\left(\mathrm{CH}_{2}-\mathrm{CH}=\mathrm{CH}_{2}\right), 81.4,74.8,68.8(\mathrm{C}-2, \mathrm{C}-3$, $\mathrm{C}-4), \quad 68.6 \quad\left(\mathrm{CH}_{2}-\mathrm{CH}=\mathrm{CH}_{2}\right), \quad 63.3 \quad(\mathrm{C}-5), \quad 26.6$ $\left((\mathrm{Ph})_{2} \mathrm{SiC}\left(\mathrm{CH}_{3}\right)_{3}\right), \quad 25.3 \quad\left(\left(\mathrm{CH}_{3}\right)_{2} \mathrm{SiC}\left(\mathrm{CH}_{3}\right)_{3}\right), \quad 18.9$ $\left((\mathrm{Ph})_{2} \mathrm{SiC}\left(\mathrm{CH}_{3}\right)_{3}\right), 18.0\left(\left(\mathrm{CH}_{3}\right)_{2} \mathrm{SiC}\left(\mathrm{CH}_{3}\right)_{3}\right)$. Anal. Calcd for $\mathrm{C}_{31} \mathrm{H}_{44} \mathrm{O}_{7} \mathrm{Si}_{2}$ (584.86): C, 63.66; H, 7.58. Found: C, 63.69; H, 7.70.

The allyloxycarbonylated product $(2.4 \mathrm{~g}, 4.1 \mathrm{mmol})$ was concentrated twice with dry THF, then dissolved in dry THF $(10 \mathrm{~mL})$ and purged with helium. After the addition of a catalytic amount of yellow tetrakis-triphenylphosphine-palladium $(0)$, the mixture was heated to $55^{\circ} \mathrm{C}$ on an oil bath. After $20 \mathrm{~min}$, when TLC (8:2 tolueneEtOAc) showed complete conversion into $9\left(R_{\mathrm{f}} 0.67\right)$, the mixture was coconcentrated with toluene. The residue was purified by column chromatography (4:6 hexane-toluene, then toluene) to yield 9 as a sirup (1.2 g, 75\%). $[\alpha]_{\mathrm{D}}=+35\left(c 1.3, \mathrm{CHCl}_{3}\right) ;{ }^{1} \mathrm{H}$ NMR $\left(\mathrm{CDCl}_{3}\right): \delta 7.65-$ 7.61, 7.46-7.38 $\left(2 \mathrm{~m}, 10 \mathrm{H}, P h_{2} \mathrm{Si}\right), 5.96-5.87(\mathrm{~m}, 1 \mathrm{H}$, $\left.\mathrm{CH}_{2}-\mathrm{CH}=\mathrm{CH}_{2}\right), 5.34-5.28,5.22-5.18(2 \mathrm{~m}$, each $1 \mathrm{H}$, $\left.\mathrm{CH}_{2}-\mathrm{CH}=\mathrm{CH}_{2}\right), 4.20\left(\mathrm{dd}, 1 \mathrm{H}, J_{2,3} 5.4, J_{3,4} 1.4 \mathrm{~Hz}, \mathrm{H}-\right.$ 3), $4.36(\mathrm{~d}, 1 \mathrm{H}, \mathrm{H}-2), 4.39-4.31,4.25-4.18(2 \mathrm{~m}$, each $\left.1 \mathrm{H}, \mathrm{CH}_{2}-\mathrm{CH}=\mathrm{CH}_{2}\right), 3.87\left(\mathrm{dd}, 1 \mathrm{H}, J_{4,5 \mathrm{a}} 2.5, J_{5 \mathrm{a}, 5 \mathrm{~b}}\right.$ $11.8 \mathrm{~Hz}, \mathrm{H}-5 \mathrm{a}), 3.76\left(\mathrm{dd}, 1 \mathrm{H}, J_{4.5 \mathrm{~b}} 3.4 \mathrm{~Hz}, \mathrm{H}-5 \mathrm{~b}\right), 1.05$ (s, $\left.9 \mathrm{H},(\mathrm{Ph})_{2} \mathrm{SiC}\left(\mathrm{CH}_{3}\right)_{3}\right), 0.87\left(\mathrm{~s}, 9 \mathrm{H},\left(\mathrm{CH}_{3}\right)_{2} \mathrm{SiC}\left(\mathrm{CH}_{3}\right)_{3}\right)$, 0.09 and $0.06\left(2 \mathrm{~s}\right.$, each $\left.3 \mathrm{H}, \mathrm{Si}\left(\mathrm{CH}_{3}\right)_{2}\right) ;{ }^{13} \mathrm{C} \mathrm{NMR}$ $\left(\mathrm{CDCl}_{3}\right): \delta 173.5(\mathrm{C}-1), 133.7\left(\mathrm{CH}_{2}-\mathrm{CH}=\mathrm{CH}_{2}\right), 135.3$ $127.8\left((\mathrm{Ph})_{2} \mathrm{SiC}\left(\mathrm{CH}_{3}\right)_{3}\right), 118.1\left(\mathrm{CH}_{2}-\mathrm{CH}=\mathrm{CH}_{2}\right), 85.6$, 74.4 and $70.5(\mathrm{C}-2, \mathrm{C}-3, \mathrm{C}-4), 71.6\left(\mathrm{CH}_{2}-\mathrm{CH}=\mathrm{CH}_{2}\right)$, $\begin{array}{llll}62.7 \quad(\mathrm{C}-5), \quad 26.7 \quad\left((\mathrm{Ph})_{2} \mathrm{SiC}\left(\mathrm{CH}_{3}\right)_{3}\right), & 25.5\end{array}$

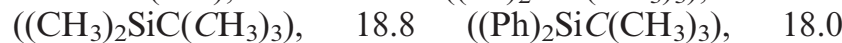
$\left(\left(\mathrm{CH}_{3}\right)_{2} \mathrm{SiC}\left(\mathrm{CH}_{3}\right)_{3}\right)$. Anal. Calcd for $\mathrm{C}_{30} \mathrm{H}_{44} \mathrm{O}_{5} \mathrm{Si}_{2}$ (540.8): C, 66.62; H, 8.20. Found: C, 66.65; H, 8.15. 


\subsection{3-O-Allyl-2-O-tert-butyldimethylsilyl-5-O-tert-butyl- diphenylsilyl-D-ribitol, 10}

To a solution of $9(700 \mathrm{mg}, 1.28 \mathrm{mmol})$ in THF $(7 \mathrm{~mL})$ was added $\mathrm{NaBH}_{4}(100 \mathrm{mg})$, and the mixture heated to $55^{\circ} \mathrm{C}$. A few drops of $\mathrm{MeOH}$ were added, and after $1 \mathrm{~h}$, TLC (9:1 toluene-EtOAc) showed complete conversion of 9 into $10\left(R_{\mathrm{f}} 0.21\right)$. The mixture was diluted with $\mathrm{MeOH}$, quenched with $\mathrm{HOAc}$ and coconcentrated with toluene. The residue was purified by column chromatography (95:5 toluene-EtOAc) to yield $\mathbf{1 0}(600 \mathrm{mg}, 86 \%)$. $[\alpha]_{\mathrm{D}}=-6\left(c \quad 0.8, \mathrm{CHCl}_{3}\right) ;{ }^{1} \mathrm{H}$ NMR $\left(\mathrm{CDCl}_{3}\right): \delta 7.66$ $7.40\left(\mathrm{~m}, \quad 10 \mathrm{H}, \quad P h_{2} \mathrm{Si}\right), \quad 5.93-5.80 \quad\left(\mathrm{~m}, \quad 1 \mathrm{H}, \quad \mathrm{CH}_{2-}\right.$ $\left.\mathrm{CH}=\mathrm{CH}_{2}\right), 5.26-5.11\left(\mathrm{~m}, 2 \mathrm{H}, \mathrm{CH}_{2}-\mathrm{CH}=\mathrm{CH}_{2}\right), 4.04$ $4.02 \quad\left(\mathrm{~m}, \quad 2 \mathrm{H}, \quad \mathrm{CH}_{2}-\mathrm{CH}=\mathrm{CH}_{2}\right), \quad 1.08 \quad(\mathrm{~s}, \quad 9 \mathrm{H}$, $\left.(\mathrm{Ph})_{2} \mathrm{SiC}\left(\mathrm{CH}_{3}\right)_{3}\right), 0.77\left(\mathrm{~s}, 9 \mathrm{H},\left(\mathrm{CH}_{3}\right)_{2} \mathrm{SiC}\left(\mathrm{CH}_{3}\right)_{3}\right), 0.06$ and $-0.06\left(2 \mathrm{~s}\right.$, each $\left.3 \mathrm{H}, \mathrm{Si}\left(\mathrm{CH}_{3}\right)_{2}\right) .{ }^{13} \mathrm{C} \mathrm{NMR}\left(\mathrm{CDCl}_{3}\right)$ : $\delta \quad 135.4-127.8 \quad\left((\mathrm{Ph})_{2} \mathrm{SiC}\left(\mathrm{CH}_{3}\right)_{3}\right), \quad 134.6 \quad\left(\mathrm{CH}_{2-}\right.$ $\left.\mathrm{CH}=\mathrm{CH}_{2}\right), \quad 116.9 \quad\left(\mathrm{CH}_{2}-\mathrm{CH}=\mathrm{CH}_{2}\right), 79.7,73.5$ and $72.6(\mathrm{C}-2, \mathrm{C}-3, \mathrm{C}-4), 70.9\left(\mathrm{CH}_{2}-\mathrm{CH}=\mathrm{CH}_{2}\right), 65.3$ and $60.9 \quad(\mathrm{C}-5, \quad \mathrm{C}-1), \quad 26.8 \quad\left((\mathrm{Ph})_{2} \mathrm{SiC}\left(\mathrm{CH}_{3}\right)_{3}\right), \quad 25.7$ $\left(\left(\mathrm{CH}_{3}\right)_{2} \mathrm{SiC}\left(\mathrm{CH}_{3}\right)_{3}\right), \quad 19.1 \quad\left((\mathrm{Ph})_{2} \mathrm{SiC}\left(\mathrm{CH}_{3}\right)_{3}\right), \quad 17.9$ $\left(\left(\mathrm{CH}_{3}\right)_{2} \mathrm{SiC}\left(\mathrm{CH}_{3}\right)_{3}\right)$. Anal. Calcd for $\mathrm{C}_{30} \mathrm{H}_{48} \mathrm{O}_{5} \mathrm{Si}_{2}$ (544.8): C, 66.13; H, 8.88. Found: C, 66.16; H, 8.84. For further analysis, a small amount of $\mathbf{1 0}$ was acetylated with $\mathrm{Ac}_{2} \mathrm{O}$ in pyridine. ${ }^{1} \mathrm{H}$ NMR $\left(\mathrm{CDCl}_{3}\right): \delta$ 7.66-7.40 (m, $\left.10 \mathrm{H}, P h_{2} \mathrm{Si}\right), 5.90-5.77\left(\mathrm{~m}, 1 \mathrm{H}, \mathrm{CH}_{2-}\right.$ $\left.\mathrm{CH}=\mathrm{CH}_{2}\right), 5.26-5.10\left(\mathrm{~m}, 2 \mathrm{H}, \mathrm{CH}_{2}-\mathrm{CH}=\mathrm{CH}_{2}\right), 5.23$ $\left(\mathrm{m}, 1 \mathrm{H}, J_{3,4} 4.2, J_{4,5 \mathrm{a}} 4.1, J_{4,5 \mathrm{~b}} 6.7 \mathrm{~Hz}, \mathrm{H}-4\right), 4.39$ (dd, $\left.1 \mathrm{H}, J_{1 \mathrm{a}, 1 \mathrm{~b}} 11.9, J_{1 \mathrm{a}, 2} 3.5 \mathrm{~Hz}, \mathrm{H}-1 \mathrm{a}\right), 4.05\left(\mathrm{dd}, 1 \mathrm{H}, J_{2,3}\right.$ $5.3 \mathrm{~Hz}, \mathrm{H}-3), 3.89$ (dd, $\left.1 \mathrm{H}, J_{5 \mathrm{a}, 5 \mathrm{~b}} 11.0 \mathrm{~Hz}, \mathrm{H}-5 \mathrm{a}\right), 3.80$ $(\mathrm{dd}, 1 \mathrm{H}, \mathrm{H}-5 \mathrm{~b}), 3.58(\mathrm{~m}, 1 \mathrm{H}, \mathrm{H}-2), 2.12$ and $2.10(2 \mathrm{~s}$, each $\left.3 \mathrm{H}, \mathrm{CH}_{3} \mathrm{CO}\right), 1.08\left(\mathrm{~s}, 9 \mathrm{H}(\mathrm{Ph})_{2} \mathrm{SiC}\left(\mathrm{CH}_{3}\right)_{3}\right), 0.77$ $\left(\mathrm{s}, 9 \mathrm{H},\left(\mathrm{CH}_{3}\right)_{2} \mathrm{SiC}\left(\mathrm{CH}_{3}\right)_{3}\right), 0.06$ and $-0.06(2 \mathrm{~s}$, each $\left.3 \mathrm{H}, \mathrm{Si}\left(\mathrm{CH}_{3}\right)_{2}\right)$.

\section{Acknowledgements}

This work was supported by the European Union program VACNET (grant ERB BIO 4CT960158).

\section{References}

1. Kamerling, J. P. Pneumococcal Polysaccharides: A Chemical View. In Molecular Biology and Mechanisms of Disease. Streptococcus pneumoniae; Thomasz, A., Ed.; Mary Ann Liebert, Inc.: New York, 2000; pp 81-114.

2. Egan, W.; Schneerson, R.; Werner, K. E.; Zon, G. J. Am. Chem. Soc. 1982, 104, 2898-2910.

3. Fleet, G. W. J.; Son, J. C. Tetrahedron 1988, 44, 26372647.

4. Zinner, H.; Voigt, H.; Voigt, J. Carbohydr. Res. 1968, 7, $38-55$.

5. Chen, S.-Y.; Jouillié, M. M. J. Org. Chem. 1984, 49, 2168 2174.

6. Shah, R. H. J. Carbohydr. Chem. 1986, 5, 139-146.

7. Baggett, N.; Buchanan, J. G.; Fatah, M. Y.; Lachut, C. H.; McCullough, K. J.; Webber, J. M. Chem. Commun. 1985, 24, 1826-1827.

8. Dho, J. C.; Fleet, G. W. J.; Peach, J. M.; Prout, K.; Smith, P. W. Tetrahedron Lett. 1986, 27, 3203-3204.

9. Ikota, N.; Hanaki, A. Chem. Pharm. Bull. 1988, 36, 1143 1146.

10. Corey, E. J.; Venkateswarlu, A. J. Am. Chem. Soc. 1972, 94, 6190-6191.

11. Masaguer, C. F.; Blériot, Y.; Charlwood, J.; Winchester, B. G.; Fleet, G. W. J. Tetrahedron 1997, 53, 15147-15156.

12. Cormier, J. F. Tetrahedron Lett. 1991, 32, 187-188.

13. Nelson, T. D.; Crouch, R. D. Synthesis 1996, 1031-1069.

14. Hanessian, S.; Lavallee, P. Can. J. Chem. 1975, 53, 29752977.

15. Guibe, F.; Saint M'leux, Y. Tetrahedron Lett. 1981, 22, 3591-3594. 